\title{
Russie : les paradoxes de la violence (Partie 3)
}

\section{Anne Le Huerou}

\section{OpenEdition}

\section{Journals}

\section{Édition électronique}

URL : http://journals.openedition.org/conflits/696

DOI : $10.4000 /$ conflits.696

ISSN : 1777-5345

Éditeur :

CCLS - Centre d'études sur les conflits lilberté et sécurité, L'Harmattan

\section{Édition imprimée}

Date de publication : 15 octobre 1998

ISSN : 1157-996X

\section{Référence électronique}

Anne Le Huerou, «Russie : les paradoxes de la violence (Partie 3) », Cultures \& Conflits [En ligne], 29-30 | automne-hiver 1998, mis en ligne le 16 mars 2006, consulté le 30 mars 2021. URL : http:// journals.openedition.org/conflits/696 ; DOI : https://doi.org/10.4000/conflits.696

Ce document a été généré automatiquement le 30 mars 2021.

Creative Commons License 


\title{
Russie : les paradoxes de la violence (Partie 3)
}

\author{
Anne Le Huerou
} entraîné dans les dernières années une " course à l'armement " d'autodéfense, favorisée par la facilité à se procurer des armes, soit en vente libre, soit provenant de la vente illégale de stocks par les militaires, et au développement rapide des sociétés proposant des services de sécurité1. Récemment par exemple, les juges ${ }^{2}$, à l'encontre desquels les menaces de mort et les agressions ont triplé ces dernières années, ont demandé à bénéficier de la loi sur le port d'arme ${ }^{3}$.

A un autre niveau, on observe le retour des Drouzhini, ces milices de quartiers composés de citoyens volontaires qui assuraient à l'époque soviétique une sorte d'îlotage. Elles sont associées aujourd'hui à l'âge d'or de la sécurité et l'on considère qu'elles assuraient au moins un maintien de l'ordre dissuasif par leur présence sur un territoire de dimensions réduites dont elles connaissaient les recoins et les habitants. Le fait qu'elles soient composées d'habitants du quartier eux-mêmes contribuait au sentiment de sécurité en même temps qu'elles participaient au contrôle social du système. Aujourd'hui, la renaissance des Drouzhini dans certains quartiers ne revêt plus que ce caractère sécuritaire " Souvent, ce ne sont pas des gens normaux, ils sont potentiellement dangereux, sans formation, et en infraction à la loi lorsqu'ils sont armés "4.

développement des milices privées et autres moyens d'autodéfense renvoie directement à la crise des organes de maintien de l'ordre, par exemple à l'incapacité de la police à défendre les premières manifestations de l'entreprenariat privé, les " coopératives " des années 1988-19905. 
question et compte tenu d'un passé encore proche où l'information était sous contrôle. Les manifestations de la violence et de la criminalité sont abondamment relayées par les médias russes, qu'il s'agisse des grands journaux moscovites, de la presse régionale ou de magazines d'informations télévisés. Les documentaires largement diffusés de $\mathrm{S}$. Govorukhine sur la grande révolution criminelle ou la Russie que nous avons perdue ou d'autres séries télévisées ${ }^{6}$, sont une illustration de ce phénomène. Assassinats commandités, règlements de compte entre groupes criminels, tueurs en série, violence domestique : l'ensemble du répertoire de la violence est présent, avec une tendance à amplifier le phénomène en juxtaposant des exemples appartenant à des registres très différents, et sur le mode du sensationnel, de la révélation, ou de l'accusation d'un haut personnage de l'Etat. Parfois, la mise en scène, dans le style anglo-saxon du docudrama est proche de la manipulation. En revanche, les analyses de fond sont très peu présentes, et l'on a le sentiment que les médias donnent à voir la violence sans la médiation d'un débat, sans plus jouer le rôle de relais d'opinion, de vecteur de l'espace public qui les avaient caractérisés pendant la perestroïka.

7 Le document du VNII cité plus haut souligne l'influence des médias et parle d'un stress collectif du crime qui " reproduit une représentation de la réalité dans la conscience de masse fabriquée non par les faits eux-mêmes mais par les témoignages à leur propos ". Ceci est mesuré plus ou moins objectivement par le taux de victimisation rapportée à la peur d'être victime. Dans un autre sondage du même organisme, les deux réponses possibles à une question sur les médias reflètent cette ambivalence entre une vision de l'information comme utilité sociale et le libre cours de la parole : $56 \%$ des personnes interrogées considèrent que " les médias doivent tout dire, même le pire "; pour $31 \%$, ils doivent " seulement exprimer des points de vue utiles à la société ". De même, " les médias ont une grande responsabilité [...]. Il n'y a presque plus que de la 'presse jaune' qui raconte n'importe quoi. C'est d'ailleurs la perestroïka qui a permis cela. Puisqu'il n'y a plus de filtre social, il faut rétablir la censure "7.

On peut retenir cette opinion, exprimée par un policier responsable du service de presse de l'UVD' à Omsk, pour rendre compte des relations entre médias et police : " On voudrait que les journalistes nous présentent bien mais ce n'est pas le cas. En plus, ils sont souvent informés avant nous et nous ne sommes pas prêts à répondre "9.

9 Sur l'attitude des médias par rapport au problème de la violence, on trouve couramment deux types d'explication : selon le premier, les faits de violence étaient objectivement tout aussi nombreux pendant la période soviétique, et la différence réside dans la manière dont les médias en rendent compte, ce qui influe directement sur les représentations de la violence et donc sur l'importance que prend ce thème dans la société : " Il y a vingt ans, un gars se faisait agresser dans la rue, puis il rentrait chez lui et voyait à la télévision un monde où tout allait bien avec des kolkhoziens souriants. Aujourd'hui, le même gars subit la même agression, il rentre chez lui et la télévision lui renvoie cent cas d'agressions semblables ". L'autre insiste sur l'augmentation objective du phénomène, les médias n'étant que le reflet de la situation réelle.

10 Ce qui est reproché aux médias, c'est de donner une image de la violence qui provoque une réaction ambiguë dans l'opinion : d'un côté, l'insistance des médias sur l'impunité des criminels renforce la crainte et aggrave la défiance vis-à-vis des institutions; de l'autre, elle renvoie des criminels une image de force et de toute puissance, physique et psychologique, notamment face à une police qui faillit à sa tâche.

11 Les perceptions de la criminalité et de la violence 
12 De fait, la société semble s'être immunisée face à la violence, ou plutôt s'en protège pour éviter de sombrer dans la panique. Mais peut-on dire que, plus profondément, elle l'accepte?

L'absence de mémoire de la violence et de débat sur la violence dans la sphère privée peut être soulignée : " Le pays s'est enfoncé dans la violence, que l'on ressent nuit et jour dans les chambres et les baraquements, mais personne n'ose discuter publiquement des questions de la violence, de la mort et du sexe (...) Dans $28 \%$ des familles seulement, les aînés parlaient aux plus jeunes des répressions, des prisons, des déportations. $11 \%$ se rappellent les conversations avec leurs parents sur la mort, $4 \%$ sur le sexe, $2 \%$ sur le suicide " ${ }^{10}$. On a le sentiment que la violence du totalitarisme est passée dans la sphère publique de la mémoire à l'histoire, mais qu'elle n'a pas fait ce chemin dans la sphère privée.

La production filmographique et littéraire russe, que l'on ne peut analyser ici en détail est une source privilégiée si l'on s'intéresse aux perceptions. Aux films " sur la violence ", qui tout en décrivant crûment les phénomènes, en parlaient en déployant une palette diversifiée de situations historiques ou socio-psychologiques, ont succédé des films " simplement violents ". Quant à la littérature, on peut distinguer entre deux tendances : d'une part le succès massif des detektivy, les romans policiers, dont une production russe, en résonance avec l'expérience vécue de la Russie actuelle, a supplanté les traductions américaines en vogue pendant la perestroïka; d'autre part une " autre littérature ", placée sous le signe du mal et qui a relégué à l'arrière plan l'humanisme d'une partie de la littérature russe et soviétique : " La nouvelle littérature oscille entre un désespoir noir et une indifférence parfaitement cynique.(...) Violence, sadisme destins brisés sont désormais des thèmes à la mode (...) On décrit toutes sortes d'avilissemenst (l'armée, la prison, les voyous), de déviances sexuelles. (...) Ce n'est plus le goulag, mais la Russie en décomposition qui devient une métaphore de la vie "11.

Les thèmes de la crise et de la décadence, assortis de la nostalgie et d'un l'appel à l'ordre, forment aujourd'hui le corps du discours, notamment parmi les intellectuels. Ceux-ci adhérent largement à la représentation d'une violence partout présente, qu'ils relient parfois à la perte de leur rôle de formation des opinions : ainsi le philosophe V. D. Gubin considère la période post-stalinienne comme une période relativement stable où la sécurité de l'individu était garantie et où les intellectuels jouaient un rôle important, par contraste avec la période actuelle, " ère de la violence de tous contre tous. La violence est devenue une norme de la vie et plus personne n'écoute les intellectuels "12. Logiquement, suivant cette hypothèse, une sortie par la construction $\mathrm{du}$ droit est peu probable, trop de couches de la population étant " lumpenisés ". Ne reste comme issue qu'une légalisation par l'Etat, lui-même corrompu, des structures maffieuses et criminelles, une sorte d'achat de la paix civile au prix de la société.

Cette attitude peut aller jusqu'à former des " théories du complot " avec un discours qui stigmatise les foyers de violence et mêle revendications séparatistes, trafics de drogues, mainmise sur les ressources du pétrole et fanatisme religieux : la région du Caucase et particulièrement la Tchétchénie, est le lieu par excellence de cette violence. Instrumentalisée par les élites locales dans les Républiques, permise par la faiblesse ou les connexions crapuleuses des autorités régionales et locales dans les autres régions, il s'agit de la menace principale.

17 Avec presque autant de force, les thèmes de la perte de la spiritualité, de la décadence morale et notamment de l'irruption de la violence et de la pornographie dans les 
médias et le circuit des cassettes vidéos, sont largement répandus: " Il y a une zombification de la société, visible dans l'augmentation des films violents à la télévision, dans la renaissance du paganisme, des sectes, la symbolique des groupes rock.[...] Aujourd'hui, le satanisme est dans les programmes de télévision "13.

Pour la génération formée au modèle soviétique, la perte de la " responsabilité sociale " des médias ou du cinéma ne parvient pas à être dépassée et laisse la porte ouverte à toutes les hypothèses et à tous les fantasmes, alimentés par les " théories du complot " qui voient derrière les processus en cours la main des sectes ou d'une cinquième colonne, et facilités par l'absence de recherches sur les relations entre médias et violence ${ }^{14}$.

Les représentations de la violence peuvent aussi s'appréhender dans la persistance d'attitudes répressives, mais aussi tolérantes vis-à-vis de certaines de ses manifestations. Dans leur enquête sur l'attitude de l'opinion face aux mauvais traitements dans l'armée, les sociologues du VTSIOM ${ }^{15}$ remarquent que si ces pratiques sont de plus en plus rejetées, notamment par les jeunes et par les couches les plus urbanisées de la société, une distinction peut être établie entre ceux qui les refusent catégoriquement et ceux qui considèrent qu'il s'agit d'un système d'éducation d'une génération par une autre, nécessaire à l'apprentissage de la vie et à la discipline.

Cette distinction s'exprime de manière encore plus nette lorsqu'il est question des groupes dit " déviants "16. Les réponses données rendent compte d'attitudes souvent franchement répressives, surtout vis-à-vis des " déviances " considérés comme l'apanage du monde occidental : homosexualité, sida, rockers. Seuls les mendiants et les alcooliques sont l'objet d'attitudes plus tolérantes. Pour les handicapés de naissance et les malades mentaux, les attitudes sont très directement héritées de la période soviétique. La dureté des termes proposés au choix des enquêtés (" liquider " ou " isoler de la société ") est un indicateur du caractère répressif qui imprègne nombre d'attitudes morales et culturelles, et la violence contenue dans les réponses n'est pas purement symbolique.

21 Dans une représentation sociale qui reste très traditionnelle, l'accroissement de la violence des jeunes (que l'on reconnait avoir toujours existé) est notamment expliquée par la disparition des institutions de contrôle et d'encadrement (pionniers, komsomols, activités peri-scolaires nombreuses et gratuites) ou l'affaiblissement de leur autorité (école). Cette explication ne mentionne pas la contrainte, et même la violence que pouvaient sécréter ces institutions ${ }^{17}$. Mais on a pu observer que la persistance de ce contrôle pouvait contenir l'apparition de la violence. Un cas qui ne doit pas conduire à des hypothèses trop rapides est celui de la violence en milieu scolaire et de l'attitude des enseignants vis-à-vis des élèves. Dans l'expérience d'une directrice d'école ${ }^{18}$, telle qu'elle nous la relate, l'école apparait comme un sanctuaire; si des violences sont à craindre, c'est essentiellement de l'extérieur, de la part d'alcooliques ou de drogués, parfois des parents ou d'anciens élèves, ce qui conduit les parents et les enseignants à demander des vigiles à l'entrée ${ }^{19} ; \mathrm{s}^{\prime} \mathrm{il}$ y a violence à l'intérieur de l'établissement, c'est entre les élèves et pas contre les enseignants; en tout état de cause, c'est l'enseignant et notamment le professeur principal qui est responsable de " l'atmosphère générale de la classe ". Si elle se dégrade, c'est lui qui en est responsable et qui est éventuellement sanctionné; la contrepartie est sinon une surveillance, tout au moins une vigilance, exercée par l'enseignant sur les élèves, y compris hors du territoire de l'école ${ }^{20}$. 
Russie, la sociét n'est pas " prete à la violence "25 : ni sur e thène de la détionation Russie, la société n'est pas " prête à la violence " 25 : ni sur le thème de la détérioration de la situation économique et sociale; ni sous la forme de mouvements d'autodéfense généralisée face à la faillite de la police; ni à propos de la situation des Russes vivant dans les autres Républiques. Mais, au-delà des indignations médiatiques ou des slogans de campagnes électorales sur les menaces dont ils sont l'objet, ce thème ne trouve dans la société, ni le sens d'un motif à prendre les armes pour les défendre, ni le sens d'une solidarité vis-à-vis des très nombreux réfugiés arrivant en Russie.

Sur le plan des valeurs et des attitudes politiques, les études, très nombreuses, portent essentiellement sur le degré de tolérance ou de préparation de la société russe à la démocratie, sur le maintien d'attitudes autoritaires, sur la mesure du " désir d'ordre " ou " d'homme fort ". Selon un sondage du VNII effectué en 1995, 45 \% des personnes interrogées accepteraient une limitation de leurs droits individuels si la défense des intérêts de l'Etat l'exigeait, 29 \% n'accepteraient en aucun cas. Dans une autre enquête sur les attitudes par rapport aux droits, $49 \%$ des personnes interrogées ${ }^{21}$ considèrent que le plus dangereux pour la société est " le non-châtiment des coupables " et $37 \%$, de " condamner des innocents".

L'aspiration autoritariste est une constante de l'analyse politique en Russie depuis plusieurs années. De nombreux sondages semblent la prouver, les intellectuels la redoutent ou la justifient ${ }^{22}$. Les réactions de l'intelligentsia libérale et démocrate aux événements d'octobre 1993 peuvent être lues de cette manière. Dans son écrasante majorité, elle a soutenu le Président Eltsine et l'assaut du Parlement, considérant le

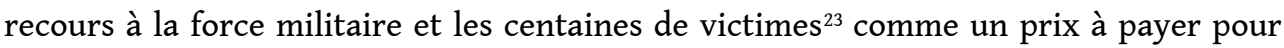
sauver la démocratie contre les " bruns-rouges ". Certains intellectuels ont été très actifs dans l'appel aux autorités à employer la force. Ils n'ont vu ni la contradiction intrinsèque de cette attitude, ni la coupure que ces événements allaient achever de provoquer entre le pouvoir et la société. Cette coupure a par exemple traversé Memorial, l'usage de la force du côté du pouvoir faisant resurgir la discussion sur les fins et les moyens, l'éthique et la politique, thèmes majeurs de la dissidence dont cette organisation est issue.

tendance à faire le choix des valeurs autoritaires ${ }^{24}$. Cette même intelligentsia fera preuve par ailleurs d'une attitude plus tolérante vis-à-vis des homosexuels ou des malades du sida que les couches moyennes de province, qui ont assisté, choquées, il est vrai sans se mobiliser, à l'assaut de la Maison blanche puis à la dissolution des soviets locaux élus démocratiquement en 1990 et auxquels elles étaient attachées.

tacite des actes de violence, ce qui peut amener les groupes qui l'utilisent à étendre leurs actions, sachant qu'ils bénéficieront, en plus de l'impunité du côté des institutions, d'une absence de résistance du côté de la société. Mais il ne s'agit pas pour autant d'adhésion, sauf peut-être pour quelques secteurs marginaux. Il s'agit plutôt d'une élévation du seuil de tolérance à la violence, due à deux éléments. D'une part, un phénomène " d'anesthésie " généralisée dans la société : lutte pour la survie, absence de perspectives, absence de débats, manque de confiance dans les institutions. D'autre part, l'existence d'un ensemble de valeurs autoritaires et répressives qui ne font pas toujours considérer la violence comme illégitime.

Le refus de la violence

Cultures \& Conflits, 29-30 | automne-hiver 1998 

chective, suggèrent que le passage à des violences nationalistes ou nostalgiques de l'empire soviétique est peu probable. Ce qui ne veut pas dire que la nostalgie de l'Union soviétique n'existe pas, ni que la " question nationale " en Russie ne soit pas cruciale. Mais les conditions qui transformeraient l'amertume ou la fierté humiliée ne sont pas réunies. S'agit-il d'un refus conscient, argumenté, ou plutôt d'un désintérêt général, d'une indifférence, qui serait en quelque sorte l'autre versant de la tolérance à la violence manifestée par ailleurs ? 65 \% des personnes interrogées en 1993 sur les conflits de l'ex-URSS considéraient que la Russie ne devait en aucun cas s'en mêler. En août 1994, 49 \% avaient la même opinion pour la Tchétchénie, censée appartenir à la Fédération de Russie. On constate aussi que le refus de la guerre se manifeste de façon abstraite : la réponse " je suis contre parce que je condamne toute guerre " vient en premier. Des réponses plus élaborées (considérant que ce conflit ne doit pas être résolu par les armes, ou que ces conséquences peuvent mener à la fin de la démocratie en Russie et marquent le retour de l'Etat vers la violence...) viennent en dernier. A Ekaterinbourg, en janvier 1995, seulement 53 \% sont préoccupés par la mort des soldats russes, et $30 \%$ par la présence de nombreux civils parmi les victimes.

31 Ces éléments peuvent contribuer à expliquer les très faibles mobilisations qui se sont manifestées lors de la guerre en Tchétchénie. Certains démocrates ont protesté, mais le caractère très politique de cette protestation n'a pas entraîné dans la rue une opinion, qui voyait d'abord en eux les responsables de réformes économiques aux conséquences dramatiques et qui n'avait pas oublié que beaucoup d'entre eux avaient approuvé l'assaut contre le Parlement.

Le Comité des mères de soldats, créé au plus fort des " mouvements informels " de la perestroïka est parmi les rares organisations à avoir conservé une capacité de mobilisation, des réseaux en province et une action continue ${ }^{28}$. Créé à la fin des années quatre-vingt, après le retour des soldats d'Afghanistan, le Comité des mères de soldats 
s'est d'abord constitué pour dénoncer les violations des droits de l'homme dans l'armée, exiger des autorités militaires des enquêtes et des explications sur les morts suspectes des appelés en temps de paix. Petit à petit, il a gagné en crédibilité face aux autorités militaires, en axant son action sur le droit. L'action a aussi porté sur le problème des incorporations abusives et sur le droit à un service alternatif, inscrit dans la Constitution mais dont la loi d'application n'a jamais été votée. Les organisations de mères de soldats, conscientes de la crise profonde de l'armée et sachant que certains militaires sont convaincus de la nécessité des réformes, se sont placées dans une approche de redéfinition des relations entre sphères civile et militaire en Russie, et ont toujours maintenu des contacts au plus haut niveau.

Il existe aujourd'hui plusieurs associations, dont la plus connue est le Comité des mères de soldats de Russie. La Fondation pour le droit des mères publie régulièrement des rapports sur les violations des droits de l'homme dans l'armée, de même que l'Organisation indépendante de Saint-Pétersbourg, plus radicale dans son rapport aux autorités civiles et militaires, et qui tente de faire échapper de nombreux jeunes appelés à l'incorporation.

Dès le début de la guerre en Tchétchénie, la mobilisation a été permanente: organisation de piquets devant la Douma, manifestations, conférences de presse, recherche des victimes et des prisonniers et publication de listes de victimes, aide aux déserteurs et aux insoumis, notamment en intervenant auprès des autorités militaires pour qu'ils ne soient pas poursuivis. L'action la plus spectaculaire a été la mobilisation de très nombreuses mères, de tous les milieux et de toutes les régions de Russie, qui sont allées jusqu'à Grozny, rechercher leurs fils prisonniers ${ }^{29}$.

En-dehors de l'expérience singulière des mères de soldats, on constate globalement une absence de mobilisation pour refuser la violence. Certes, de très nombreuses organisations se placent sur le terrain des violations des droits de l'homme ${ }^{30}$, de la condition des prisonniers, ou militent pour l'abolition de la peine de mort. Dans une société très peu mobilisée, ces organisations manifestent leur refus de la violence, leur attachement à la démocratie et leur volonté de construire une société civile en Russie essentiellement par le canal de l'information (bulletins réguliers, recueil de témoignages et documents, conférences de presse). Elles sont composées en grande majorité d'intellectuels et se diffusent peu dans la société. Au contraire, leurs désaccords politiques dès qu'il s'agit de se positionner par rapport au pouvoir et une tendance à ne voir d'espoir possible que dans le soutien de la communauté internationale (renouant en cela avec la démarche des dissidents) renforcent la coupure avec l'opinion publique.

On observe la même absence de mobilisation pour des acteurs qui explicitement se réclament de la violence, par exemple le groupe fasciste de Barkachov, Russkij Poriadok (L'ordre russe). Au-delà de l'organisation elle-même, qui rassemble des militants souvent jeunes, le rejet viscéral de ce type d'orientation politique est manifeste. Lorsque l'un des responsables de ce mouvement a déclaré début 1995 qu'il aimerait " tirer une balle dans le dos " du défenseur des droits de l'homme Sergueï Kovalev, il a été attaqué en justice ${ }^{31}$. La société russe, malgré le sentiment d'insécurité massif que l'on a analysé, et qui peut facilement être repris par un discours sécuritaire, malgré aussi les attitudes répressives mentionnées plus haut, n'est pas pour autant extrémiste.

Conclusion : un nouveau paradigme? 
La sortie du système soviétique, avec un Etat qui contrôlait à la fois l'économie, la vie sociale, le système politique et la nation constitue évidemment un bouleversement considérable. De ce point de vue, la violence qui traverse le pays peut être considérée comme la poursuite, sous forme de réactions en chaîne, de la secousse principale au sommet. Selon les " terrains ", cela se traduit soit en " trop-plein ", soit en vide. En trop-plein avec la montée des identités nationales et culturelles jusque dans les revendications des minorités à l'intérieur de chaque nouvel Etat de l'ancienne URSS, ainsi qu'avec les transformations brutales du marché. En vide, si l'on considère la faiblesse de l'espace du politique et du social en Russie.

Le caractère post-totalitaire de la violence en Russie réside aussi dans la privatisation progressive d'un phénomène auparavant principalement du ressort de l'Etat. La montée de la criminalité, et notamment de la criminalité économique à la fin du régime soviétique, est aussi un signe, en creux, de l'épuisement du totalitarisme.

L'explication de la montée de la violence dans le monde contemporain par absence ou carence de l'Etat ${ }^{32}$, semble pouvoir s'appliquer à la Russie. La décomposition et la faiblesse de l'Etat favorisent ainsi l'apparition d'espaces où la violence se manifeste. La violence d'en bas révèle l'incapacité de l'Etat à assurer la protection et la sécurité de la population tandis que la légitimité de la violence d'en haut (assaut du Parlement en 1993, Tchétchénie) est largement contestée. La difficulté d'appréhender la nature et le fonctionnement du pouvoir en Russie, y compris dans son recours à la violence, peut le faire qualifier de " coton "33, par opposition au " mur " qu'il constituait à l'époque soviétique, auquel on se heurtait très durement mais qui renvoyait une réponse. C'est ici, la non lisibilité des processus " en haut " conjuguée au caractère formel de la démocratie et à la faiblesse de l'espace public, qui sont sources d'insécurité et de violences.

41 Encore capable d'autoritarisme mais affaibli, l'Etat est aux mains de centres de pouvoir diffus et informels qui, faute de légitimité, ont souvent recours à la force pour s'imposer, mais jamais plus que partiellement et temporairement. L'image générale est celle d'une " tendance à l'autodéfense de tous les individus et de tous les groupes sociaux " 34 qui engendre de la violence mais la contient la plupart du temps dans des affrontements de " basse intensité ", pour utiliser le vocabulaire stratégique ou " moléculaires " pour reprendre l'expression d'Hanz-Magnus Enzensberger quant à la violence urbaine.

L'on assisterait alors, avec le processus de privatisation de la violence que l'on a décrit plus haut à la fin de " l'Etat wébérien ". Une autre hypothèse est également possible, celle d'un nouveau type d'Etat qui gère seulement certaines formes de la "violence légitime ". La Russie contemporaine se situerait alors " ailleurs " du point de vue de l'Etat, et il faudrait partir de la description de la situation observée pour avancer vers une autre conception, soit reliée à une " tradition russe "35, soit d'un type nouveau. Ce débat dépasse l'analyse du problème de la violence et ramène au problème de l'analyse de la Russie, entre le renvoi constant à une spécificité essentielle et la soumission aux catégories habituelles de la sociologie ou de la science politique, en faisant abstraction de l'histoire et de la culture. Le débat n'est pas propre à ce pays mais il y est exacerbé par l'histoire des discussions et des passions politiques autour de la soviétologie ${ }^{36}$.

Pour de nombreux observateurs, c'est l'introduction brutale de l'économie de marché qui aurait ouvert la voie à la violence ouverte. Cette hypothèse est étroitement liée à la précédente, la criminalisation de l'économie se traduisant par un affaiblissement de 
l'Etat encore plus grand, voire par la création d'un " Etat criminel " : " L'arrivée du marché a entraîné une dureté dans les relations humaines et une augmentation de la criminalité jusqu'au niveau de la vie quotidienne. Plus la violence se développe, plus s'affaiblit l'immunité contre elle, l'intolérance à la violence dans la société. " ${ }^{37}$.

Une version plus optimiste de ce raisonnement voit dans la violence un problème général d'adaptation aux transformations du système, un phénomène de transition, qu'il s'agisse de la construction du marché, des transformations géopolitiques de l'espace ex-soviétique, ou des mentalités individuelles qui sont plus ou moins capables de surmonter les conditions d'instabilité et d'indétermination de la vie collective et individuelle de la nouvelle période.

Par ailleurs, deux discours, en apparence contradictoires, ne cessent de se juxtaposer. Le premier exprime un sentiment d'insécurité, très au-delà du niveau réel de violence, et particulièrement intolérant aux incivilités, dont la définition dépasse les manifestations de violence physiques mais renvoie aussi à des comportements déviants vécus comme autant d'agressions. Ce sentiment est d'autant plus vif qu'il est mesuré à l'aune de la tranquillité et de la sécurité dont chacun semblait bénéficier auparavant. Et un deuxième discours traduit une tendance largement répandue, essentiellement parmi les auteurs et les analystes, à considérer le problème en terme de civilisation des mœurs inachevée. En seraient responsables, d'une part les soixante dix ans d'un système qui, par le principe même de son idéologie et par la terreur, aurait éliminé la civilisation jusque dans chaque individu, et d'autre part un " caractère national russe ", dont peut témoigner le niveau élevé de violences interpersonnelles et domestiques. Ces explications relèvent souvent d'une sociologie spontanée des mentalités et de la culture, même si, plus sérieusement, elles peuvent en appeler au parallèle tenté part Norbert Elias entre les cultures russe et allemande, l'une et l'autre, historiquement incapables de se dominer sans " homme fort ", et caractérisées par le " développement d'un Surmoi fragmentaire et irrégulier " dont les trous ouvrent la voie à la violence ${ }^{38}$. Dans une perspective plus sociologique, on peut interpréter en termes de norme de comportement admise dans la société la dureté qui prévaut dans les interactions quotidiennes. Ce type de violence interpersonnelle doit être alors distingué des incivilités, assimilées au " hooliganisme " et aux dégradations, auxquelles la société est particulièrement intolérante, ce qui complique le raisonnement proposé par Hugues Lagrange ${ }^{39}$, qui note que la grande sensibilité aux incivilités se manifeste dans les pays qui ont atteint un haut niveau de civilisation des moeurs.

Si l'on tente une référence aux théories classiques, la Russie d'aujourd'hui serait plus proche de celle de Hobbes que de celle de Locke : le renoncement à la violence comme expression de " civilité " et non de lâcheté, paraît en effet au plus loin de la réalité observée dans un pays où la faiblesse, qu'elle soit physique ou psychologique, est méprisée. En revanche, dans une " société [qui] ne produit rien d'autre que des relations de pouvoir et de subordination "40, les concepts d'allégeance et de protection qui définissent les termes du " pacte hobbesien " du renoncement à la violence de tous contre tous, semblent correspondre pour partie à la privatisation de la violence que l'on a décrite. Un support pourrait lui être donné par le terme très en vogue en Russie de Krysha (toit) qui désigne tout type de protection et peut être considéré comme un élément essentiel de la vie économique, sociale et politique actuelle du pays ${ }^{41}$. violence selon Hobbes : " Ce qui empêche les lois naturelles de la sociabilité d'opérer, 
c'est précisément l'absence de garantie dans l'état de nature. Il y a violence quand personne ne sait à quoi s'attendre, quand personne ne peut plus compter sur rien, quand tout peut arriver, quand font défaut les règles qui rendent prévisibles les comportements et fondent les attentes de réciprocité dans les interactions " ${ }^{42}$. Bien que ne lui étant pas destinée, cette phrase décrit de manière très juste la situation russe. Un autre point de vue lui fait écho: pendant la période soviétique, des " règles du jeu " existaient, non pas tant celles du système formel que celles, tacites, que tout le monde utilisait, y compris dans le règlement des conflits, y compris dans la violence. " On savait où s'arrêter. Si on perdait, on perdait. Aujourd'hui, c'est un processus sans fin, on peut prolonger les conflits indéfiniment, et il n'y a pas de limites dans les moyens. On peut tuer quelqu'un pour presque rien. La seule solution serait que la justice fonctionne, mais dans l'état actuel, il faudrait que la moitié de la population devienne magistrat... "43.

Il est plus difficile encore d'expliquer l'absence que la présence de violence. Il est vrai que les conditions du passage à la violence ne sont pas réunies : la continuité des élites, notamment en province, et les caractéristiques sociales d'une population urbanisée et éduquée, où ne se rencontrent pas les " masses de jeunes déclassés au chômage, sans perspective, qui sont à la base des conflits dans le Caucase ou dans les Balkans "44, apportent un début d'explication, que confortent les désertions massives de jeunes appelés pendant la guerre en Tchétchénie, et l'état de démoralisation de l'armée. La patience ${ }^{45}$, décrite non seulement comme un trait historique et culturel du peuple russe, mais comme une stratégie face aux mutations en cours, peut aussi constituer un facteur favorable au non-passage à la violence.

En revanche, on pourrait voir dans la violence des institutions telles que l'armée ou la prison la manifestation cachée d'une violence sociale qui ne dit pas son nom, masquée par un discours sur l'héritage totalitaire de la Russie. C'est peut-être là que se révèlent le plus fortement l'anomie et la subjectivité impossible. La relation ambiguë qu'entretient vis-à-vis de ces manifestations la société elle-même serait une confirmation de cette hypothèse.

50 Mais, mieux vaut, au-delà de ces hypothèses trop fragiles, s'interroger sur la signification de la violence. Deux types de questionnement se posent : le premier est de savoir ce qui, dans une société, à un moment de son histoire, est désigné comme violence; le second est d'examiner si elle y est légitime ou illégitime et comment elle est refusée ou tolérée. En Russie, la violence est désignée comme criminalité quand elle est instrumentale, comme cruauté lorsqu'elle est objet de jugements moraux, pour partie héritiers de la dissidence, et semble relever de la banalité lorsqu'elle affecte les rapports humains quotidiens.

51 Aujourd'hui, des normes et des systèmes de représentations opposés coexistent dans un même espace, en semblant parfois appartenir à des temps différents: les comportements criminels liés aux activités économiques; les valeurs humanistes des " résistants " qui nomment la violence, la dénoncent ou cherchent à la prévenir ; les normes traditionnelles des responsables institutionnels en charge de la punir. Ses expressions sont nombreuses, mais éclatées, désarticulées et sans relais politique. Ce qui peut à la fois expliquer que la société les tolère, éventuellement les justifie, mais aussi qu'elle ne bascule pas dans la violence généralisée.

52 L'absence de cohérence des systèmes de représentations face à la violence et l'absence de cohérence dans la société contribuent à la confrontation directe des expressions et 
des représentations du phénomène, sans la médiation d'un espace public qui ouvre le débat et pose les enjeux. Une sortie de la violence actuelle supposerait l'existence d'un tel espace, ce qui signifierait sortir de la décomposition - au risque de voir surgir une violence sociale ou politique qui pourrait alors constituer la " maladie infantile " de la recomposition de débats et de rapports sociaux et politiques.

\section{NOTES}

1. Au 1er avril 1995, on comptait en Russie 6605 entreprises de protection et de sécurité et vingt-six mille personnes avaient reçu une licence leur permettant d'exercer ces fonctions. Sans compter les innombrables sociétés proposant des alarmes, portes grillagés, serrures, etc.

2. Les Nouvelles de Moscou, 18 avril 1996

3. La loi sur les armes et instructions supplémentaires du MVD en date d'avril 1995 réglemente l'autodéfense et les conditions de délivrance d'un port d'arme. Elle est très peu restrictive.

4. Entretien avec O. R., journaliste à l'hebdomadaire Novoe Obozrenie, Omsk, 25 avril 1996 ;

5. Mark Galeotti, " Perestroïka, perestrelka, pereborka : policing Russia in a time of change ", Europe-Asia Studies, vol. 45, n 5, 1993, p. 769-786.

6. Comme Kriminal'naâ Rossiâ, (La Russie criminelle) diffusée sur la chaîne indépendante NTV le samedi à une heure de grande écoute.

7. Entretien avec I. K. de l'Ecole supérieure de la police, Omsk, 23 avril 1996.

8. Direction régionale du ministère de l'Intérieur.

9. Entretien le 25 avril 1996. En réponse à ce souci, le service a créé son propre hebdomadaire, Kriminal'naïa Khronika (Chronique criminelle), destiné à informer objectivement la population de la ville des faits survenus et de l'activité des forces de l'ordre, à publier des statistiques et des avis de recherches, aussi bien que les programmes de télévision et des romans policiers en feuilleton.

10. Ûri Levada, Entre le passé et l'avenir, l'Homme soviétique ordinaire : enquête, Paris, Presses de la Fondation Nationale des Sciences politiques, 1993, p. 129.

11. Viktor Erofeev, Les Fleurs du mal russe : anthologie de la nouvelle littérature russe, Paris, Albin Michel, 1997, p.15.

12. V. D. Gubin, " Russkaâ kultura i fenomen nasiliâ " [La culture russe et le phénomène de la violence] in Rossijsko-germanskij dialog : nasilie v posttotalitarnyh obšcestvah (Dialogue germano-russe : la violence dans les sociétés post-totalitaires), Voprosy filosofii, $n^{\circ}$ 5, 1995, p. 3-47.

13. Entretien avec I. K., professeur de criminologie à l'Ecole supérieure de la police, Omsk, 23 avril 1996.

14. Un article récent tente d'introduire un début de réflexion sur le sujet à partir des débats occidentaux, Kirill A., " Ot nasiliâ v kino k nasiliû "kak v kino" ? " [De la violence au cinéma à la violence "comme au cinéma" ?] in Sociologiceskie issledovaniâ, $\mathrm{n}^{\circ} 2$, 1996, p. 35-40. 
15. Ûri Levada, L'homme soviétique ordinaire, op. cit., chap. 5.

16. Résultats de sondages du VTSIOM, parus et analysés dans Ûri Levada, L'homme soviétique ordinaire, op. cit., dans " Škola zestokosti : sovietskie lûdi o nasilii " [L'école de la cruauté : les soviétiques face à la violence], Celovek, 1992, nº 2, p. 17-25.

17. On peut, pour ce qui concerne l'école, renvoyer au film Cucelo (L'épouvantail), réalisé en 1982 par Rolan Bykov. Au-delà de l'intérêt et de la qualité du film lui-même, il est caractéristique que toute une génération, scolarisée au début des années quatrevingt, ait été "nourrie" avec ce film qui devait l'édifier sur la cruauté et la violence dont peuvent être capables les enfants, sans remettre en cause l'institution.

18. Entretien avec O. I. S., directrice d'école, 29 avril 1996.

19. Selon les cas et les lieux, il s'agit de policiers affectés par le commissariat du quartier, de policiers embauchés en renfort financés par les parents, le plus souvent de parents qui se cotisent pour embaucher un vigile privé.

20. Un incident à titre d'exemple : le vol du carnet de notes par une élève voulant cacher ses mauvaises notes entraîne une punition collective par l'enseignante. Les autres élèves, pour se venger, suivent l'élève auteur du vol jusque chez elle après les cours, et insultent la famille, "taggent" les murs de l'immeuble, etc. La directrice a considéré l'enseignante comme responsable de l'incident : elle aurait dû prévoir la réaction des élèves et prévenir l'expédition punitive. Elle a obligé le professeur à aller avec toute la classe voir la famille pour lui présenter des excuses collectives.

21. Sondage VNII, 1995.

22. Parmi une très abondante littérature, voir par exemple Igor M. Klâmkin, Viktor V. Lapkin V. I. Pantin, "' Mezdu avtoritarizmom i demokratiej " ([Entre autoritarisme et démocratie] in Politiceskie issledovanniâ (Polis), n 2, 1995, p. 56-96 ; Igor M. Klâmkin, " Kakoj avtoritarnyj rezim vozmozen segodnâ v Rossii " [Quel régime autoritaire est possible en Russie aujourd'hui ?] dans Polis, $\mathrm{n}^{\circ}$ 5, 1993, p. 50-54 ; Matthew Wyman, " Russian political culture : evidence from public opinion surveys " dans The Journal of Communist Studies and Transition Politics, vol. 10, n 1, mars 1994, p. 25-54 ; Grigorii Vainshtein, " The authoritarian idea in the public conscious and political life of contemporary Russia ", Ibid., vol. 11, n³, sept. 1995, p. 272-285.

23. Dont le nombre doit se situer entre les chiffres officiels de cent quarante-huit morts et les milliers annoncés par l'opposition.

24. Comme l'analyse en détails Maria Ferretti, " De l'engouement pour la liberté à l'apologie de l'autoritarisme : la métamorphose de l'intelligentsia démocratique russe ", dans De Russie et d'ailleurs, feux croisés sur l'histoire. Mélanges offerts à Marc Ferro, Paris, Institut d'études slaves, 1995, p. 151-165.

25. Cf. L.Y. Gozman, " Psihologiâ peremena " [La psychologie du changement], Rossijsko-germanskij dialog : nasilie v posttotalitarnyh obšcestvah in (Dialogue germano-russe : la violence dans les sociétés post-totalitaires), Voprosy filosofii, $n^{\circ} 5$, 1995, p. 3-47.

26. Segodnâ, 19 janvier et 19 février 1995 notamment.

27. A Ekaterinburg, dans l'Oural. Ural'skij fond social'nyh issledovannii, Ural : politika, Èkonomika, pravo, (Oural : politique, économie, droit), n (10), 1995.

28. Cf. Françoise Daucé, " les mouvements de mères de soldats à la recherche d'une place dans la société russe ", Revue d'Etudes Comparatives Est-Ouest, n², juin 1997. 29. Parfois, il ne s'agissait pas de prisonniers, mais de soldats stationnés dans leurs unités, que les mères ont aidés à déserter. 
30. Memorial, une des premières associations informelles créées pendant la perestroïka pour rendre justice aux victimes du stalinisme, et dont de nombreux militants sont eux-mêmes passés par les camps, publie un hebdomadaire sur les droits de l'homme Express Khronika, et participe aux mobilisations pour la défense des droits de l'homme et contre la guerre en Tchétchénie.

31. Une nouvelle émission a été consacrée à cette "affaire" sur le thème de savoir s'il fallait ou non diffuser de tels propos, dans des termes plus virulents que ceux des débats français sur la banalisation ou la martyrisation du Front national.

32. Voir notamment Philippe Delmas, Le bel avenir de la guerre, Paris, Gallimard, 1995.

33. Interview de l'ancien dissident et président de la Fondation Glasnost, S.Grigoriants, Paninter, mai 1997.

34. Boris Slavnyj, " Grazdanskoe obšcestvo ili grazdanskaâ vojna ? " [Société civile ou guerre civile ?] op. cit.

35. Voir l'article de Claudio Sergio Ingerflöm, " Russie impériale, Russie soviétique : quelques thèmes pour une histoire politique de longue durée ", [Retour sur l'URSS], Historiens et géographes, $n^{\circ} 351$, décembre 1995, p. 145-158.

36. Voir notamment Wladimir Berelowitch, "La soviétologie après le putsch : vers une guérison ", [Back in USSR : représentations de l'Union soviétique], Politix, $\mathrm{n}^{\circ} 18,2 \mathrm{e}$ trim. 1992, p. 7-20.

37. Entretien avec Vitali Kvashis.

38. Norbert Elias, Norbert Elias par lui-même, Paris, Fayard, 1991.

39. Hughes Lagrange, la civilité à l'épreuve : crime et sentiment d'insécurité, Paris, PUF, 1995.

40. Idem.

41. Vladimir Shlapentokh, " Russia : privatisation and illegalization of social and political life ", The Washington Quarterly,19 (1), hiver 1996, p. 65-85.

42. Yves Michaud, " Les violences de l'histoire ", Esprit, mai 1994, p. 11.

43. Entretien avec D. V., politologue et expert, 26 avril 1996.

44. Andrej Fadin, " Vlast' cerez krov' : put' naverh (Socialnyj smysl' postsovetskyh vojn) ", op. cit.

45. Vladimir Shlapentokh, "Russian patience : a reasonable behavior and a social strategy ", Archives européennes de sociologie, vol. 36, n² 2, 1995, p. 247-280.

INDEX

Index géographique : Russie

Mots-clés : mafias et crime organisé, violence 